\title{
Safety-Related Regulatory Actions and Risk Factors for Anticancer Drugs in Japan
}

\author{
Hiroki Nakayama ${ }^{1}\left[\right.$. Naoki Matsumaru ${ }^{2} \cdot$ Katsura Tsukamoto $^{2}$
}

Published online: 26 November 2018

C) Springer Nature Switzerland AG 2018

\begin{abstract}
Introduction The approval of anticancer drugs in Japan has increased to meet high medical demand. To maximize the benefits of anticancer drugs, adverse drug reactions (ADRs) must be properly managed. However, in some cases, clinically significant safety issues are detected after launch, and safety-related regulatory actions (SRRAs) are implemented.

Objectives We aimed to determine the characteristics of SRRAs for anticancer drugs approved in Japan and to identify factors related to the drug development and regulatory approval process associated with the occurrence of an SRRA.

Methods We defined an SRRA as the issuance of a 'Yellow Letter', 'Blue Letter', or an official notification by the Ministry of Health, Labor and Welfare. Anticancer drugs approved in Japan as new active ingredients from April 2004 to July 2016 were analyzed using publicly available information. The Kaplan-Meier survival curve was plotted to estimate the probability of the occurrence of an SRRA, and the Cox proportional hazards model was used to identify risk factors associated with the occurrence of an SRRA. Independent variables were selected using backward/forward stepwise selection according to Akaike's Information Criterion.

Results An SRRA was implemented for 38 of 63 anticancer drugs. Approximately $70 \%$ of SRRAs occurred within 2 years after approval, and the median time between approval and the occurrence of an SRRA was 1.6 years (interquartile range 0.94-2.4). No Yellow Letter was issued during the follow-up period; however, one Blue Letter was issued for 'acute lung injury and interstitial pneumonia' for sorafenib. According to official notifications, 'clinically significant adverse reactions' was the most revised section of package inserts (62\%). The probability of an SRRA at the 1-, 2- and 3-year follow-up was 15.9\% (95\% confidence interval [CI] 6.4-24.4\%), 41.3\% (95\% CI 27.8-52.3\%), and 56.8\% (95\% CI 41.8-68.0\%), respectively. Monoclonal antibodies were associated with a low risk of occurrence of an SRRA (hazard ratio [HR] 0.29, $p=0.019$ ), while the large number of patients in pivotal studies (per 100 patients) was associated with a high risk of occurrence (HR 1.07, $p=0.012$ ).

Conclusions The high-risk period for the occurrence of an SRRA for anticancer drugs in Japan was within 2 years after approval. Among the factors related to the drug development and regulatory approval process, anticancer drugs in the form of non-monoclonal antibodies, and whose pivotal studies included a large number of patients, were more likely to be associated with an SRRA. Postmarketing follow-up should therefore be carefully performed, especially in the first 2 years after approval and for non-monoclonal antibody anticancer drugs. Moreover, postmarketing follow-up is crucial, even if large-scale pivotal studies for regulatory approval have already been performed.
\end{abstract}

Electronic supplementary material The online version of this article (https://doi.org/10.1007/s40290-018-0260-8) contains supplementary material, which is available to authorized users.

Hiroki Nakayama

hiroki.nakayama@astellas.com

1 Healthcare Policy and CSR, Astellas Pharma Inc., 2-5-1, Nihonbashi-Honcho, Chuo-ku, Tokyo 103-8411, Japan

2 Global Regulatory Science, Gifu Pharmaceutical University, 1-25-4, Daigakunishi, Gifu 501-1196, Japan

\section{Key Points}

The high-risk period for the occurrence of a safetyrelated regulatory action (SRRA) for anticancer drugs in Japan was within 2 years after approval.

Among the factors related to the drug development and regulatory approval process, monoclonal antibodies were associated with a low risk of occurrence of an SRRA, while the large number of patients in pivotal studies (per 100 patients) was associated with a high risk of occurrence of an SRRA. 


\section{Introduction}

Most clinical studies are limited in size, target narrow welldefined populations, and primarily focus on establishing efficacy [1]. As such, safety data are not always sufficiently established at the time of approval of new drugs. In particular, anticancer drugs are known to cause a variety of adverse drug reactions (ADRs), and clinically significant safety issues are sometimes first detected after a drug has already launched. A previous study on all drug types reported that anticancer drugs are significantly associated with the occurrence of a safety-related regulatory action (SRRA) [2].

Several developments related to the drug development and regulatory approval process of anticancer drugs have been made. First, advances in the development of molecular techniques has led to the discovery of tumor-specific molecular targets. Therefore, monoclonal antibodies that can logically be applied to cancers with tumor-specific molecular targets have emerged as standard therapeutic agents for cancer [3]. These drugs are generally well tolerated and are associated with a lower risk of serious ADRs than other anticancer drugs [4]. Second, anticancer drugs with an orphan drug designation have been increasingly approved in Japan in recent years. These drugs are approved based on limited clinical evidence, such as in studies with a small sample size or non-randomized or non-controlled designs compared with non-orphan drugs [5]. Third, drug lag, a time delay in the approval of a drug compared with other countries such as the United States (US), is a recognized social issue in Japan because the unavailability of new drugs due to an approval lag negatively impacts a population's health. As such, the Ministry of Health, Labor and Welfare (MHLW) and Pharmaceuticals and Medical Devices Agency (PMDA) have taken several measures to reduce the approval lag [6]. Although an approval lag for anticancer drugs still exists, the lag peaked in 2002 and has since declined [7], suggesting that Japanese patients now have earlier access to new anticancer drugs.

In Japan, health authorities or regulatory agencies are likely to implement an SRRA when they observe clinically significant safety issues resulting from the use of a drug. We hypothesized that characteristics of the drug development and regulatory approval process, including the above movements, may affect the implementation of an SRRA for anticancer drugs. To test this hypothesis, we aimed to identify factors related to the drug development and regulatory approval process associated with the occurrence of an SRRA for anticancer drugs approved in Japan. We also attempted to determine the characteristics of SRRAs, such as the high-risk period for the occurrence of an SRRA for anticancer drugs. To our knowledge, this is the first study to reveal the characteristics of SRRAs for anticancer drugs, which are known to cause a variety of ADRs compared with other drugs, in Japan.

\section{Methods}

Important safety information that needs swift communication is released through the Dear Healthcare Professional Letters of Emergent Safety Communications, known as a 'Yellow Letter', or Dear Healthcare Professional Letters of Rapid Safety Communications, known as a 'Blue Letter'. The Yellow Letter documents emergent and significant safety information about drugs, while the Blue Letter documents information that may not be emergent but should nevertheless be rapidly reported to alert healthcare professionals [8]. Additionally, an official notification issued by the MHLW requires immediate package insert (PI) revision and prompt dissemination of information to healthcare professionals $[2,9,10]$. In this study, we defined an SRRA as the issuance of a Yellow Letter, Blue Letter, or an official notification.

\subsection{Samples}

This study targeted all anticancer drugs approved in Japan as new active ingredients for systemic therapy to treat malignant tumors from April 2004 to July 2016. Figure 1 shows the flowchart of sample selection. Of the 74 anticancer drugs approved in Japan during the study period, the following drugs were excluded: (1) drugs whose PMDA review reports were not available; (2) drugs approved for benign tumors, palliative therapy or supportive therapy, including adjuvant therapy; and (3) drugs that were not approved for comparable indications in the US because an approval lag between the US and Japan was selected as an independent variable for multivariate analysis.

\subsection{Data Collection}

Data on anticancer drugs approved in Japan were obtained from lists of approved products, review reports, PIs, and a summary of the new drug application (NDA) dossier available from the PMDA website. We also collected the following data on SRRAs from the 'The Yellow Letter/Blue Letter' and 'Revisions of PRECAUTIONS in drug package inserts' pages of the PMDA website, and identified the occurrence of an SRRA: (1) name of the anticancer drug; (2) date of SRRA implementation; (3) ADRs; (4) type of SRRA; and (5) the revised sections of PIs related to safety: 'warnings', 'careful administration', 'important precautions', or 'clinically significant adverse reactions'. ADRs were categorized by system organ class (SOC) according to the Common 


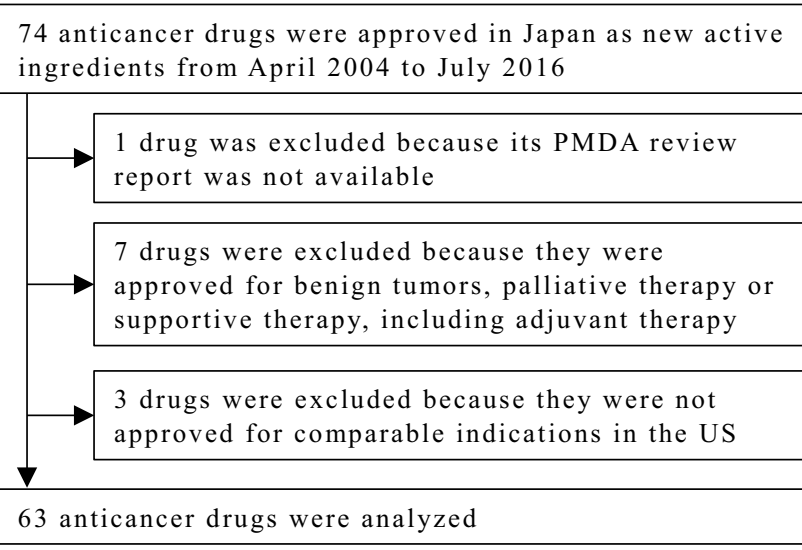

Fig. 1 Study sample selection. PMDA pharmaceuticals and medical devices agency

Terminology Criteria for Adverse Events version 4.03 [11]. Information related to reviews by the US FDA was collected from approval letters, review reports, and PIs available from the FDA website. All data generated or analyzed during this study are included in this article and its supplementary information file (electronic supplementary Table S1).

\subsection{Data Analysis}

A Kaplan-Meier survival curve was plotted to estimate the probability of the occurrence of an SRRA, and the Cox proportional hazards model was used to identify risk factors associated with the occurrence of an SRRA. Independent variables were selected using backward/forward stepwise selection according to Akaike's Information Criterion [12]. The model satisfied the proportional hazard assumption. All statistical analyses were performed using EZR software version 1.36, with $\alpha=0.05$ as the statistically significant threshold [13]. The cut-off date was set for 31 July 2018 to ensure at least 2 years of follow-up for all drugs analyzed because most SRRAs were implemented within 2 years of approval, as shown in this study.

\subsection{Independent Variables in the Cox Proportional Hazards Model}

Based on the reasons stated below, we hypothesized that the potential factors related to the drug development and regulatory approval process that may affect the occurrence of an SRRA were 'drug characteristics', 'clinical development', and 'regulatory status', for which we examined a total of six independent variables in the Cox proportional hazards model.

We examined two variables among drug characteristics: 'monoclonal antibody' and 'first-in-class'. We speculated that monoclonal antibodies may be associated with a low risk of an SRRA because these drugs are generally more specific towards their target than most small molecules, and are therefore generally well tolerated and associated with a low risk of serious ADRs [4]. In contrast, we speculated that first-in-class drugs would be associated with a high risk of occurrence of an SRRA because the safety information on the class effects of these drugs may be sparse at approval. We defined a first-in-class drug as a drug that acts on new targets regardless of whether they are small molecules or biologics. According to a previous study, first-in-class biologicals are associated with a significantly higher risk of occurrence of an SRRA than later approved drugs [14].

For clinical development, we examined the 'approval lag between the US and Japan' and 'number of patients in pivotal studies' as independent variables. We speculated that an approval lag between the US and Japan is likely to be associated with a low risk of an SRRA because a previous study reported that safety data collected from foreign countries before the launch of a drug in Japan reduced the probability of the occurrence of an SRRA [2,9]. The approval lag was calculated by subtracting the approval date in the US from that in Japan. We specifically investigated the approval lag between Japan and the US because the approval lag for anticancer drugs between Japan and the US is longer than that between Japan and the European Union (EU) [15], and a larger number of new molecular entities are approved in the US than in the EU [16]. Additionally, we predicted that the large number of patients in pivotal studies might be associated with a low risk of occurrence of an SRRA because the inclusion of more patients in pivotal studies for anticancer drugs means more safety data can be acquired. Pivotal studies were defined as clinical trials that were reviewed by the PMDA as being the most crucial trial for their evaluation. We used the safety analysis set to determine the number of patients in pivotal studies. If there were multiple pivotal studies for one NDA, all of these studies were considered pivotal studies.

We also examined the regulatory statuses 'orphan drug designation in Japan' and 'accelerated approval in the US' as independent variables. We predicted that orphan anticancer drugs may be associated with a low risk of an SRRA. The number of patients treated with an orphan drug is usually much lower than those treated with a non-orphan drug. As a consequence, the chances of encountering clinically significant ADRs during clinical use are not very high, and SRRAs may take longer to implement for orphan drugs than non-orphan drugs [17]. In Japan, the MHLW can designate a drug that satisfies the following criteria as an orphan drug on receiving an application for orphan drug designation: (1) the number of patients in Japan who may use the drug is less than 50,000 ; (2) the drug is indicated for the treatment of serious diseases, including difficult-to-treat diseases, with 
high medical needs; and (3) there is a theoretical rationale for use of the product for the target disease and the development plan is appropriate [18]. In contrast, accelerated approval may be associated with a high risk of occurrence of an SRRA, given that the approval of drugs under accelerated approval is based on limited clinical evidence, making it more likely that clinically significant ADRs are detected after launch. A previous study targeting orphan drugs reported that orphan drugs approved by accelerated approval are associated with a higher risk of occurrence of an SRRA [17]. In Japan, accelerated approval by the conditional early approval system was started in 2017 [19]. However, before this system was implemented, anticancer drugs were similarly approved based on limited clinical evidence [5]. This is because pivotal studies that were reviewed by the FDA to support accelerated approval were also submitted in Japan as pivotal studies to support approval under bridging strategies, and global clinical trials as development strategies. Based on these circumstances, we selected 'accelerated approval in the US' as an independent variable instead of 'conditional early approval system in Japan'.

\section{Results}

We analyzed a total of 63 anticancer drugs. Figure 2 shows the number of anticancer drugs approved in Japan between 2004 and 2016; the number of anticancer drugs approved in 2015-2016 was more than three times greater than that approved in 2004-2006.

Table 1 presents the characteristics of anticancer drugs analyzed in this study. Among the types of drugs, monoclonal antibodies and other anticancer drugs comprised $21 \%$ and $79 \%$ of the drugs analyzed, respectively. The median number of patients in pivotal studies was 410 [interquartile range (IQR) 250-760]. An SRRA was implemented for 38 of the 63 anticancer drugs (60\%).

Table 2 lists the anticancer drugs for which SRRAs were implemented. Approximately $70 \%$ of SRRAs occurred within 2 years after approval. The median time between approval and SRRA was 1.6 years (IQR 0.94-2.4). No Yellow Letter was issued during the follow-up period; however, one Blue Letter was issued for 'acute lung injury and interstitial pneumonia' for sorafenib. According to official notifications, 'clinically significant adverse reactions' was the most revised section of PIs (62\%). The 'warnings' section of PIs was only revised for crizotinib, to add 'fulminant hepatitis' as safety information. There was no clear trend in the categories of ADRs of SRRAs according to SOC.

Figure 3 shows the Kaplan-Meier analysis of the probability of the occurrence of an SRRA. The probability of an SRRA at the 1-, 2- and 3-year follow-up was 15.9\% (95\% confidence

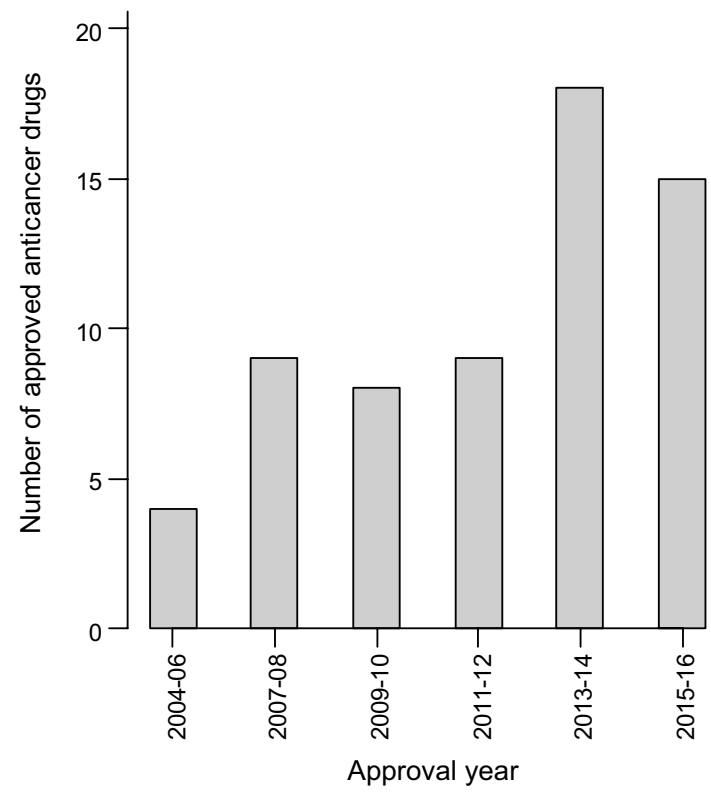

Fig. 2 Number of anticancer drugs approved in Japan between 2004 and 2016

interval [CI] 6.4-24.4\%), 41.3\% (95\% CI 27.8-52.3\%), and $56.8 \%$ (95\% CI 41.8-68.0\%), respectively.

Table 3 presents the risk factors of anticancer drugs associated with the occurrence of an SRRA. Monoclonal antibodies were significantly associated with a low risk of occurrence of an SRRA (hazard ratio [HR] 0.29, $p=0.019$ ). In contrast, the large number of patients in pivotal studies (per 100 patients) was significantly associated with a high risk of occurrence of an SRRA (HR 1.07, $p=0.012$ ).

\section{Discussion}

Anticancer drugs are known to cause a variety of ADRs, some of which can lead to SRRAs. We aimed to identify the high-risk period among anticancer drugs for the occurrence of an SRRA. Additionally, we also aimed to identify the risk factors associated with the occurrence of an SRRA among various variables related to the drug development and regulatory approval process. Approximately $70 \%$ of SRRAs occurred within 2 years after approval, suggesting that the high-risk period for the occurrence of an SRRA for anticancer drugs in Japan is within 2 years of drug approval. The rate of the occurrence of an SRRA in this study was higher than that reported by a previous study on biologicals in the US and EU [14]. The probability of the occurrence of an SRRA in this study was also higher than that reported by a past study targeting all drug types in Japan, and biologicals and orphan drugs in the US and EU [2, 14, 17]. These findings may suggest that anticancer drugs approved in Japan 
Table 1 Characteristics of analyzed anticancer drugs

\begin{tabular}{|c|c|c|c|}
\hline Factor & Item & $N(\%)$ & Median (IQR) \\
\hline & Drugs (total) & 63 & \\
\hline & Drugs with an SRRA & & \\
\hline & Yes & $38(60)$ & \\
\hline & No & $25(40)$ & \\
\hline \multirow[t]{6}{*}{ Drug characteristics } & Type of drug & & \\
\hline & Monoclonal antibody & $13(21)$ & \\
\hline & Other anticancer drug & $50(79)$ & \\
\hline & First-in-class & & \\
\hline & Yes & $21(33)$ & \\
\hline & No & $42(67)$ & \\
\hline \multirow[t]{2}{*}{ Clinical development } & Approval lag between the US and Japan, years & & $2.6(0.94-4.0)$ \\
\hline & Number of patients in pivotal studies, per 100 patients & & $4.1(2.5-7.6)$ \\
\hline \multirow[t]{6}{*}{ Regulatory status } & Orphan drug designation in Japan & & \\
\hline & Yes & $36(57)$ & \\
\hline & No & $27(43)$ & \\
\hline & Accelerated approval in the US & & \\
\hline & Yes & $22(35)$ & \\
\hline & Other & $41(65)$ & \\
\hline
\end{tabular}

$I Q R$ interquartile range, $S R R A$ safety-related regulatory action are likely to be associated with a high risk of occurrence of an SRRA.

We identified two risk factors of anticancer drugs that were associated with the occurrence of an SRRA. Monoclonal antibodies were significantly associated with a low risk of occurrence of an SRRA. The fact that cancer cells share many similarities with normal host cells makes it challenging to achieve high levels of selective cytotoxicity. Monoclonal antibodies are engineered with the advantage of specificity, to act as 'targeting missiles' towards cancer cells [3]. Therefore, these drugs are generally well tolerated and are associated with a lower risk of serious ADRs than other anticancer drugs [4]. Our results support these commonly accepted notions, indicating the validity of our analysis.

Contrary to our prediction, the large number of patients in pivotal studies was significantly associated with a high risk of occurrence of an SRRA. This discrepancy may reflect the fact that our findings demonstrate the exposure of patients to the drugs after launch, rather than the accumulated safety data at the time of approval. In clinical development of anticancer drugs, particularly for drugs indicated for non-major cancers, the target number of patients in pivotal studies may be designed based on the number of patients with the target cancers. Therefore, a smaller number of patients in pivotal studies likely reflects a smaller number of patient exposures after launch. A previous study reported that a large number of patient exposures may result in a higher risk of occurrence of an SRRA [2], which supports our findings. Our result also indicates that the increase in the patient population from narrow, well-defined populations in clinical trials to those in actual clinical practice may raise the risk of an SRRA. Therefore, postmarketing follow-up is crucial, even if largescale pivotal studies for regulatory approval have already been performed. A Risk Management Plan (RMP) for new drugs is one measure started in Japan in 2013 for effective postmarketing follow-up. The RMP aims to document appropriate management, methods to minimize and characterize known and potential risks for each drug, and to share the information among stakeholders [20]. Efficient data collection from clinical trials is crucial for creating an effective RMP for anticancer drugs, especially drugs indicated for non-major cancers.

An example of an anticancer drug with identified factors associated with the occurrence of an SRRA is cabazitaxel, a microtubule inhibitor. The NDA for cabazitaxel was submitted in Japan in July 2013 and approved in July 2014 for the treatment of prostate cancer. The pivotal study in the NDA was a foreign phase III study and the safety analysis sets comprised 742 patients, which was larger than the median number of patients in pivotal studies. In December 2014, an official notification was issued by the MHLW to add bone marrow depression to the 'important precautions' section of the PI [21]. Another example is sorafenib, a multikinase inhibitor that targets several serine/threonine and receptor tyrosine kinases. The NDA for sorafenib was submitted in June 2006 based on a foreign phase III study as a pivotal study, and approval was granted in January 2008 for the treatment of unresectable or metastatic renal cell carcinoma. The safety analysis sets of the pivotal study comprised 
Table 2 Anticancer drugs with a safety-related regulatory action

\begin{tabular}{|c|c|c|c|c|}
\hline Generic name & $\begin{array}{l}\text { Time from approval } \\
\text { to an SRRA (years) }\end{array}$ & ADR & Type of SRRA & $\begin{array}{l}\text { Revised section } \\
\text { of package } \\
\text { insert }\end{array}$ \\
\hline Letrozole & 1.07 & $\begin{array}{l}\text { Osteoporosis } \\
\text { Fracture }\end{array}$ & ON & $\begin{array}{l}\text { IP } \\
\text { IP }\end{array}$ \\
\hline Temozolomide & 2.46 & Interstitial pneumonia & ON & CSAR \\
\hline Bortezomib & 1.92 & Ileus & ON & CSAR \\
\hline Pemetrexed sodium hydrate & 3.73 & $\begin{array}{l}\text { Infections } \\
\text { Toxic epidermal necrolysis and oculomucocutaneous } \\
\text { syndrome (Stevens-Johnson syndrome) }\end{array}$ & ON & $\begin{array}{l}\text { CSAR } \\
\text { CSAR }\end{array}$ \\
\hline Bevacizumab & 2.45 & Interstitial pneumonia & ON & CSAR \\
\hline \multirow[t]{3}{*}{ Erlotinib hydrochloride } & 1.61 & $\begin{array}{l}\text { Oculomucocutaneous syndrome (Stevens-Johnson } \\
\text { syndrome), toxic epidermal necrolysis (Lyell syn- } \\
\text { drome) and erythema multiforme }\end{array}$ & ON & CSAR \\
\hline & & Gastrointestinal perforation & & CA, CSAR \\
\hline & & Corneal perforation and corneal ulcer & & IP, CSAR \\
\hline Nelarabine & 4.81 & Rhabdomyolysis & ON & CSAR \\
\hline Sorafenib tosilate & 0.90 & Acute lung injury and interstitial pneumonia & $\mathrm{BL}, \mathrm{ON}$ & IP, CSAR \\
\hline Ibritumomab tiuxetan & 2.75 & Infections & $\mathrm{ON}$ & CSAR \\
\hline Sunitinib malate & 1.45 & Disseminated intravascular coagulation syndrome & ON & CSAR \\
\hline Cetuximab & 1.68 & $\begin{array}{l}\text { Heart failure } \\
\text { Severe diarrhea }\end{array}$ & $\mathrm{ON}$ & $\begin{array}{l}\text { CSAR } \\
\text { CSAR }\end{array}$ \\
\hline Thalidomide & 1.45 & Teratogenicity & $\mathrm{ON}$ & IP \\
\hline Nilotinib hydrochloride hydrate & 1.95 & Tumor lysis syndrome & ON & CSAR \\
\hline Dasatinib hydrate & 2.76 & Pulmonary arterial hypertension & $\mathrm{ON}$ & CSAR \\
\hline Everolimus & 1.17 & $\begin{array}{l}\text { Infections } \\
\text { Hyperglycemia and development or exacerbation of } \\
\text { diabetes mellitus } \\
\text { Pulmonary embolism and deep vein thrombosis } \\
\text { Acute respiratory distress syndrome }\end{array}$ & $\mathrm{ON}$ & $\begin{array}{l}\text { IP, CSAR } \\
\text { CSAR } \\
\text { CSAR } \\
\text { CSAR }\end{array}$ \\
\hline Panitumumab & 2.95 & Hypomagnesemia & $\mathrm{ON}$ & CSAR \\
\hline Lenalidomide hydrate & 0.74 & $\begin{array}{l}\text { Cerebral infarction and transient ischemic attacks } \\
\text { Infections } \\
\text { Hepatic function disorder and jaundice } \\
\text { Bone marrow depression }\end{array}$ & $\mathrm{ON}$ & $\begin{array}{l}\text { CSAR } \\
\text { CSAR } \\
\text { CSAR } \\
\text { CSAR }\end{array}$ \\
\hline Temsirolimus & 1.97 & $\begin{array}{l}\text { Mucositis oral } \\
\text { Anemia, thrombocytopenia, leukopenia, neutropenia, } \\
\text { and lymphopenia }\end{array}$ & $\mathrm{ON}$ & $\begin{array}{l}\text { CSAR } \\
\text { CSAR }\end{array}$ \\
\hline Bendamustine hydrochloride & 1.49 & Infections & ON & IP, CSAR \\
\hline Azacitidine & 1.26 & Interstitial lung disease & ON & CSAR \\
\hline Eribulin mesylate & 4.82 & $\begin{array}{l}\text { Oculomucocutaneous syndrome (Stevens-Johnson } \\
\text { syndrome) and erythema multiforme }\end{array}$ & $\mathrm{ON}$ & CSAR \\
\hline Crizotinib & 1.78 & $\begin{array}{l}\text { Fulminant hepatitis } \\
\text { Bradycardia }\end{array}$ & ON & $\begin{array}{l}\text { W, IP, CSAR } \\
\text { IP, CSAR }\end{array}$ \\
\hline Degarelix acetate & 1.10 & Shock and anaphylaxis & ON & CSAR \\
\hline Axitinib & 1.32 & Heart failure & ON & CSAR \\
\hline Carmustine & 3.77 & Neurological symptoms & ON & IP \\
\hline Pazopanib hydrochloride & 0.18 & $\begin{array}{l}\text { Thrombotic microangiopathy } \\
\text { Pancreatitis }\end{array}$ & ON & $\begin{array}{l}\text { CSAR } \\
\text { CSAR }\end{array}$ \\
\hline Regorafenib hydrate & 0.58 & Thrombocytopenia & ON & CSAR \\
\hline Afatinib maleate & 2.26 & Acute pancreatitis & ON & CSAR \\
\hline Enzalutamide & 0.58 & Thrombocytopenia & ON & CSAR \\
\hline Abiraterone acetate & 0.58 & $\begin{array}{l}\text { Hypokalemia } \\
\text { Thrombocytopenia } \\
\text { Rhabdomyolysis }\end{array}$ & $\mathrm{ON}$ & $\begin{array}{l}\text { CA, IP, CSAR } \\
\text { CSAR } \\
\text { CSAR }\end{array}$ \\
\hline
\end{tabular}


Table 2 (continued)

\begin{tabular}{|c|c|c|c|c|}
\hline Generic name & $\begin{array}{l}\text { Time from approval } \\
\text { to an SRRA (years) }\end{array}$ & ADR & Type of SRRA & $\begin{array}{l}\text { Revised section } \\
\text { of package } \\
\text { insert }\end{array}$ \\
\hline Cabazitaxel acetonate & 0.47 & Bone marrow depression & ON & IP \\
\hline Nivolumab & 1.20 & $\begin{array}{l}\text { Excessive immunoreaction } \\
\text { Myasthenia gravis and myositis } \\
\text { Colitis and severe diarrhea }\end{array}$ & $\mathrm{ON}$ & $\begin{array}{l}\text { IP } \\
\text { CSAR } \\
\text { CSAR }\end{array}$ \\
\hline Bosutinib hydrate & 1.86 & Reactivation of hepatitis B virus & ON & IP \\
\hline Vemurafenib & 2.14 & Acute kidney injury & $\mathrm{ON}$ & IP, CSAR \\
\hline Lenvatinib mesylate & 0.67 & Hemorrhage & $\mathrm{ON}$ & CA, IP, CSAR \\
\hline Pomalidomide & 0.36 & Hepatic function disorder and jaundice & $\mathrm{ON}$ & CSAR \\
\hline Ipilimumab & 2.53 & Myositis & $\mathrm{ON}$ & CSAR \\
\hline Trabectedin & 0.56 & Cardiac dysfunction & $\mathrm{ON}$ & CA, IP, CSAR \\
\hline
\end{tabular}

$A D R$ adverse drug reaction, $B L$ Blue Letter, $C A$ careful administration, CSAR clinically significant adverse reactions, $I P$ important precautions, $O N$ official notification, $S R R A$ safety-related regulatory action, $W$ warnings

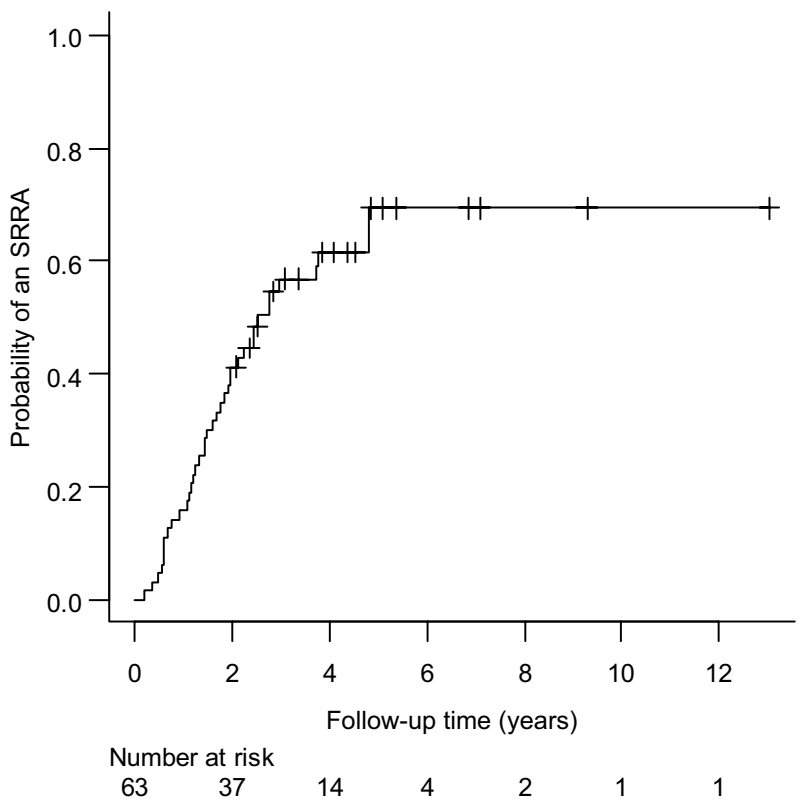

Fig. 3 Kaplan-Meier analysis of the probability of the occurrence of an SRRA. SRRA safety-related regulatory action

Table 3 Risk factors of anticancer drugs associated with the occurrence of a safety-related regulatory action

\begin{tabular}{llc}
\hline Independent variable & Adjusted HR (95\% CI) & $p$ value \\
\hline Type of drug & & \\
$\quad$ Monoclonal antibody & $0.29(0.10-0.82)$ & 0.019 \\
$\quad$ Other anticancer drug & 1.00 & \\
Number of patients in pivotal & $1.07(1.01-1.13)$ & 0.012 \\
$\quad$ studies, per 100 patients & & \\
Number of anticancer drugs & 63 & \\
Number of events & 38 & \\
\hline
\end{tabular}

$C I$ confidence interval, $H R$ hazard ratio
768 patients, which was larger than the median number of patients in pivotal studies. A Blue Letter was issued for acute lung injury and interstitial pneumonia. Additionally, an official notification was issued by the MHLW in December 2008 to add acute lung injury and interstitial pneumonia to the 'important precautions' and 'clinically significant adverse reactions' sections of the PI [22].

There are some limitations associated with this study. First, its retrospective nature limits the strength of conclusions that can be drawn from the results. Second, the sample size of this study was relatively small. The accumulation of more samples is needed to obtain more reliable results. Third, the independent variables were selected from factors related to drug characteristics, clinical development, and regulatory status because the objectives of this study were to identify factors related to the drug development and regulatory approval process associated with the occurrence of an SRRA for anticancer drugs approved in Japan. Independent variables related to postmarketing may also be associated with the occurrence of an SRRA. Notwithstanding these limitations, we are confident that our analyses are sufficient for achieving the purpose of this study.

\section{Conclusions}

The high-risk period for the occurrence of an SRRA for anticancer drugs in Japan was within 2 years after approval. Among factors related to the drug development and regulatory approval process, anticancer drugs in the form of nonmonoclonal antibodies, and whose pivotal studies included a large number of patients, were more likely to be subjected to an SRRA. Postmarketing follow-up should therefore be carefully performed, especially in the first 2 years after approval and for non-monoclonal antibody anticancer drugs. 
Moreover, postmarketing follow-up is crucial, even if largescale pivotal studies for regulatory approval have already been performed.

Acknowledgements The authors express their gratitude to Katsuya Nakano for his review of the study from a regulatory affairs viewpoint.

\section{Compliance with Ethical Standards}

Funding The authors received no financial support for the research, authorship, and/or publication of this article.

Conflicts of interest Hiroki Nakayama is an employee of Astellas Pharma Inc. Naoki Matsumaru and Katsura Tsukamoto declare no conflicts of interest.

Ethical approval This article does not contain any studies with human participants or animals performed by any of the authors.

\section{References}

1. Mol PG, Arnardottir AH, Motola D, Vrijlandt PJ, Duijnhoven RG, Haaijer-Ruskamp FM, et al. Post-approval safety issues with innovative drugs: a European cohort study. Drug Saf. 2013;36(11):1105-15.

2. Fujikawa M, Ono S. Analysis of safety-related regulatory actions for new drugs in Japan by nature of identified risks. Pharm Med. 2017;31(5):317-27.

3. Coulson A, Levy A, Gossell-Williams M. Monoclonal antibodies in cancer therapy: mechanisms, successes and limitations. West Indian Med J. 2014;63(6):650-4.

4. Catapano AL, Papadopoulos N. The safety of therapeutic monoclonal antibodies: implications for cardiovascular disease and targeting the PCSK9 pathway. Atherosclerosis. 2013;228(1):18-28.

5. Nakayama $\mathrm{H}$, Tsukamoto $\mathrm{K}$. Unique characteristics of regulatory approval and pivotal studies of orphan anticancer drugs in Japan. Investig New Drugs. 2018;36(4):702-8.

6. Ueno T, Asahina Y, Tanaka A, Yamada H, Nakamura M, Uyama Y. Significant differences in drug lag in clinical development among various strategies used for regulatory submissions in Japan. Clin Pharmacol Ther. 2014;95(5):533-41.

7. Maeda H, Kurokawa T. Recent trends for drug lag in clinical development of oncology drugs in Japan: does the oncology drug lag still exist in Japan? Int J Clin Oncol. 2015;20(6):1072-80.

8. Pharmaceuticals and Medical Devices Agency. The Yellow Letter/Blue Letter. 2018. https://www.pmda.go.jp/english/safety/infoservices/drugs/esc-rsc/0001.html. Accessed 16 Nov 2018.
9. Yamada T, Kusama M, Hirai Y, Arnold F, Sugiyama Y, Ono S. Analysis of pharmaceutical safety-related regulatory actions in Japan: do tradeoffs exist between safer drugs and launch delay? Ann Pharmacother. 2010;44(12):1976-85.

10. Ishiguro C, Misu T, Iwasa E, Izawa T. Analysis of safety-related regulatory actions by Japan's pharmaceutical regulatory agency. Pharmacoepidemiol Drug Saf. 2017;26(11):1314-20.

11. National Cancer Institute. Common Terminology Criteria for Adverse Events (CTCAE) v4.03. 2010. https://ctep.cancer.gov/ protocolDevelopment/electronic_applications/ctc.htm\#ctc_40. Accessed 16 Nov 2018.

12. Akaike $\mathrm{H}$. Information theory and an extension of the maximum likelihood principle. In: Petrov BN, Caski F, editors. Second international symposium on information theory. Budapest: Akademiai Kiado; 1973. p. 267-81.

13. Kanda Y. Investigation of the freely available easy-to-use software 'EZR' for medical statistics. Bone Marrow Transplant. 2013;48(3):452-8.

14. Giezen TJ, Mantel-Teeuwisse AK, Straus SM, Schellekens H, Leufkens HG, Egberts AC. Safety-related regulatory actions for biologicals approved in the United States and the European Union. JAMA. 2008;300(16):1887-96.

15. Yonemori K, Hirakawa A, Ando M, Hirata T, Yunokawa M, Shimizu C, et al. The notorious "drug lag" for oncology drugs in Japan. Investig New Drugs. 2011;29(4):706-12.

16. Tsuji K, Tsutani K. Approval of new drugs 1999-2007: comparison of the US, the EU and Japan situations. J Clin Pharm Ther. 2010;35(3):289-301.

17. Heemstra HE, Giezen TJ, Mantel-Teeuwisse AK, de Vrueh RL, Leufkens HG. Safety-related regulatory actions for orphan drugs in the US and EU: a cohort study. Drug Saf. 2010;33(2):127-37.

18. Ministry of Health, Labour and Welfare. Overview of orphan drug/medical device designation system. (in Japanese) 2015. http://www.mhlw.go.jp/stf/seisakunitsuite/bunya/0000068484 .html. Accessed 15 Aug 2018.

19. Ministry of Health, Labour and Welfare. Implementation of a conditional early approval system for pharmaceutical products. 2017. https://www.pmda.go.jp/files/000222439.pdf. Accessed 28 Sept 2018.

20. Ministry of Health, Labour and Welfare. Implementation of the "Risk Management Plan". Pharmaceuticals and Medical Devices Safety Information No. 300. 2013. https://www.pmda.go.jp/files /000153064.pdf\#page $=5$. Accessed 28 Sept 2018 .

21. Pharmaceuticals and Medical Devices Agency. review report for cabazitaxel (in Japanese). 2014. http://www.pmda.go.jp/drugs /2014/P201400085/780069000_22600AMX00751_A100_1.pdf. Accessed 28 Sept 2018.

22. Pharmaceuticals and Medical Devices Agency. Review report for sorafenib (in Japanese). 2008. http://www.pmda.go.jp/drugs /2008/P200800005/630004000_22000AMX00014000_A100_1. pdf. Accessed 28 Sept 2018. 